\title{
On the Elzaki transform and its applications in fractional free electron laser equation
}

\author{
Yudhveer Singh \\ Amity Institute of information \\ Technology, \\ Amity University, Rajasthan, \\ Jaipur, India \\ email: yudhvir.chahal@gmail.com

\section{Sunil Kundu} \\ Department of Mathematics, \\ Jaipur National University, \\ Jaipur Rajasthan, India \\ email: kundu0716@gmail.com
}

\section{Vinod Gill}

Department of Mathematics, Govt.P.G. college Hisar, Haryana, India email: vinod.gill08@gmail.com

\section{Devendra Kumar}

Department of Mathematics, University of Rajasthan, Jaipur, Rajasthan, India email: devendra.maths@gmail.com

\begin{abstract}
This article is devoted to study Elzaki transform and its applications in Free Electron Laser equation involving Hilfer-Prabhakar fractional derivative. We derive formula of Elzaki transform for HilferPrabhakar derivative and its regularized version. The solution of Free Electron Laser equation involving Hilfer-Prabhakar fractional derivative of fractional order is presented in terms of Mittag-Leffler type function. Furthermore, we find the application of the generalized Hilfer-Prabhakar derivative in linear partial differential equation and some problems of Mathematical Physics.
\end{abstract}

2010 Mathematics Subject Classification: 26A33, 42B10, 33E12, 34A08, 44A35 Key words and phrases: Hilfer-Prabhakar fractional derivative, free electron laser equation, Elzaki transform, Mittag-Leffler function, convolution 


\section{Introduction}

In past, few years fractional calculus (integration and differentiation of arbitrary order) have received considerable attention to solve the mathematics, engineering and mathematical physics problems $[5,6,7,8,9,10,11,12,13,15$, $16,17,18,19,20,21]$. Hilfer-Prabhakar operator is a powerful tool to generalize the linear and non-linear fractional order differential equations. Prabhakar integral in generalized form of earlier versions and plays a key role to examine the Hilfer-Prabhakar fractional derivative problems using integral transform method.

In the literature of fractional differentiations and integrations there are several integral transforms like Laplace, Fourier, Mellin, Sumudu to name but a few. A new integral transform namely Elzaki transform [2] which is a modified form of classical Laplace and Sumudu transform and has some good features. Elzaki transform has been efficiently used to solve the integral equations and differential equations in fractional calculus. Using of Elzaki transform is also beneficial as it can solve a class of differential equation which are not solved by Sumudu transform.

The main objective of this article is to introduce the formulae for Elzaki transform and apply it to solve Hilfer-Prabhakar fractional derivative and its regularized version. Taking inspiration from these works our purpose is to find the solution of Cauchy problems of fractional order by using Elzaki transform. Furthermore, we discuss here the importance of Elzaki transform associated with Hilfer-Prabhakar fractional derivatives to solve free electron laser type integro-differential equation. The motivation of this paper is to encourage further investigation of the potential applications of this branch of mathematics.

\section{Basic definition}

In this section, we study some important basic definition related to fractional calculus and Elzaki transform to understand the further results, lemmas and applications.

Definition 1 Elzaki transform [2, 3] of function $\mathrm{g}(\mathrm{t})$ introduced by Tarig $M$. Elzaki is defined as

$$
p \int_{0}^{\infty} e^{-\frac{t}{p}} g(x, t) d t=\mathbf{E}[g(x, t)]=T(x, p), t>0, p \in\left(-\tau_{1}, \tau_{2}\right) .
$$


Elzaki transform of first order partial derivative is defined as

$$
\mathbf{E}\left[\frac{\partial g}{\partial t}(x, t)\right]=\frac{T(x, p)}{p}-p \cdot g(x, 0) .
$$

Now, we find relation between Laplace and Elzaki transform,

Let $\mathbf{f}(\mathrm{t}) \in A=\left\{\mathbf{f}(\mathrm{t}): \exists \mathrm{M}, \tau_{1}, \tau_{2}>0\right.$ such that $|\mathrm{f}(\mathrm{t})|<M \mathrm{e}^{|\mathrm{t}| / \tau_{i}}$ if $\left.\mathrm{t} \in(-\mathfrak{i})^{\mathfrak{j}} \times[0, \infty)\right\}$ and $\mathrm{F}(\mathrm{s})$ be the Laplace transform, the Elzaki transform $\mathrm{T}(\mathrm{u})$ is given by

$$
\mathrm{T}(\mathrm{u})=\mathrm{uF}\left(\frac{1}{\mathrm{u}}\right) .
$$

Also, the Laplace transform and the Elzaki transform [4] must coincide at $\mathrm{u}=1$ and we can write $\mathrm{T}(1)=\mathrm{F}(1)$.

Definition 2 (Hilfer derivative). Let $\mu \in(0,1), v \in[0,1], g \in \mathrm{L}^{1}[\mathrm{a}, \mathrm{b}]$, $-\infty \leq \mathrm{a}<\mathrm{t}<\mathrm{b} \leq \infty, \mathrm{f} * \mathrm{~K}_{(1-v)(1-\mu)} \in A C^{1}[\mathrm{a}, \mathrm{b}]$. Then

$$
D_{a^{+}}^{\mu, v} g(t)=\left(I_{a^{+}}^{v(1-\mu)} \frac{d}{d t} I_{a^{+}}^{(1-v)(1-\mu)} g\right)(t)
$$

is the Hilfer fractional derivative [7] of order $\mu$.

Definition 3 Regularized of Hilfer derivative [7] of same order $\mu$ is written as

$$
D_{0^{+}}^{\mu, v} g(t)-\frac{t^{-\mu} g\left(0^{+}\right)}{\Gamma(1-\mu)}={ }^{C} D_{0+}^{\mu} g(t) .
$$

Definition 4 Prabhakar introduced the generalized Mittag-Leffler function [8, 14] in the following form

$$
\mathrm{E}_{\alpha, \beta}^{\gamma}(\mathrm{z})=\sum_{\mathrm{k}=0}^{\infty} \frac{\Gamma(\gamma+\mathrm{k})}{\Gamma(\gamma) \Gamma(\alpha \mathrm{k}+\beta)} \frac{\mathrm{z}^{\mathrm{k}}}{\mathrm{k} !},
$$

where $\alpha, \beta, \gamma \in \mathbb{C}$ and $\mathbb{R}(\alpha)>0$.

The generalization of Hilfer derivative with a more general integral operator with kernel [5]

$$
t^{\mu-1} E_{\rho, \mu}^{\gamma}\left[\omega t^{\rho}\right]=e_{\rho, \mu, \omega}^{\gamma}(t), t \in \mathbb{R}, \rho, \mu, \omega, \gamma \in \mathbb{C}, \text { with } \mathbb{R}(\mu), \mathbb{R}(\rho)>0 .
$$

The well known Prabhakar integral is expressed in the similar way, replacing kernel by function and is defined as follows [5]. 
Definition 5 (Prabhakar integral) Let $\mathrm{L}^{1}[0, \mathrm{~b}], 0<\mathrm{t}<\mathrm{b} \leq \infty$. The Prabhakar integral is written as

$$
E_{\rho, \mu, \omega, 0^{+}}^{\gamma} f(t)=\int_{0}^{t}(t-y)^{\mu-1} E_{\rho, \mu}^{\gamma}\left[\omega(t-y)^{\rho}\right] f(y) d y=\left(f * e_{\rho, \mu, \omega}^{\gamma}\right)(t),
$$

where $\rho, \mu, \omega, \gamma \in \mathbb{C}$ with $\mathbb{R}(\rho), \mathbb{R}(\mu)>0$.

Definition 6 (Hilfer-Prabhakar derivative) The Hilfer-Prabhakar derivative of $\mathrm{g}(\mathrm{t})$ of order $\mu$ denoted by $\mathrm{D}_{\rho, \omega, 0^{+}}^{\gamma, \mu, \nu} \mathrm{g}(\mathrm{t})$ is defined as

$$
D_{\rho, \omega, 0^{+}}^{\gamma, \mu, v} g(t)=\left(E_{\rho, v(1-\mu), \omega, 0^{+}}^{-\gamma v} \frac{d}{d t}\left(E_{\rho,(1-\gamma)(1-\mu), \omega, 0^{+}}^{-\gamma(1-\gamma)} g\right)\right)(t),
$$

where $\mu \in(0,1), \nu \in[0,1]$, and $\gamma, \omega \in \mathbb{R}, \rho>0$, and $\mathrm{E}_{\rho, 0, \omega, 0^{+}}^{0} \mathrm{~g}=\mathrm{g}$.

Definition 7 (Regularized version of Hilfer-Prabhakar derivative) For $\mathrm{g} \in$ $A C^{1}[0, b], 0<t<b<\infty, \mu \in(0,1), v \in[0,1], \omega, \gamma \in \mathbb{R}, \rho>0$, the regularized version of Hilfer-Prabhakar fractional derivative of $\mathrm{f}(\mathrm{t})$ denoted by $\mathbf{c}_{\mathbf{D}_{\rho, \omega, 0^{+}}}^{\gamma, \mu} \mathbf{f}(\mathbf{t})$ is defined as

$$
c_{D_{\rho, \omega, 0^{+}}}^{\gamma, \mu} g(t)=\left(E_{\rho, v(1-\mu), \omega, 0^{+}}^{-\gamma v} E_{\rho,(1-\gamma)(1-\mu), \omega, 0^{+}}^{-\gamma(1-v)} \frac{d}{d t} g\right)(t),
$$

also,

$$
E_{\rho, \mu, \omega, 0^{+}}^{\gamma} E_{\rho, v, \omega, 0^{+}}^{\sigma} g(x)=E_{\rho, \mu+\gamma, \omega, 0^{+}}^{\gamma+\sigma} g(x) .
$$

\section{Elzaki transform of fractional derivatives}

In this section we introduce formula of Elzaki transform of Prabhakar integral to study Elzaki transform of Hilfer-Prabhakar fractional derivative and its regularized version.

Lemma 1 The Elzaki transform of Prabhakar integral is given by

$$
E\left[e_{\rho, \mu, \omega}^{\gamma}(t)\right]=p^{\mu+1}\left[1-\omega p^{\rho}\right]^{-\gamma},
$$

for $\mu \in(0.1), \omega, \gamma \in \mathbb{R}$ and $\rho>0$. 
Proof. The equation (4) can be written as

$$
E_{\rho, \mu}^{\gamma}\left(\omega t^{\rho}\right)=\sum_{k=0}^{\infty} \frac{\Gamma(\gamma+k)}{\Gamma(\gamma) \Gamma(\rho k+\mu)} \frac{\left(\omega t^{\rho}\right)^{k}}{k !} .
$$

Taking Elzaki transform of Prabhakar integral using (5) and (10), we have $\mathbf{E}\left[e_{\rho, \mu, \omega}^{\gamma}(t)\right]=\mathbf{E}\left[\sum_{k=0}^{\infty} t^{\mu-1} \frac{\Gamma(\gamma+k)}{\Gamma(\gamma) \Gamma(\rho k+\mu)} \frac{\left(\omega t^{\rho}\right)^{k}}{k !}\right]$

$=\mathbf{E}\left[\sum_{k=0}^{\infty} t^{\rho k+\mu-1} \frac{\Gamma(\gamma+k)}{\Gamma(\gamma) \Gamma(\rho k+\mu)} \frac{\omega^{k}}{k !}\right]$

$$
\begin{gathered}
=p^{\mu+1} \sum_{k=0}^{\infty} \frac{\Gamma(\gamma+k)}{\Gamma(\gamma)} \frac{\left(\omega p^{\rho}\right)^{k}}{k !} \\
=p^{\mu+1}\left[1-\omega p^{\rho}\right]^{-\gamma} .
\end{gathered}
$$

Which is the Elzaki transform formula of Prabhakar integral. We use this result to solve Free Electron Laser equation involving Prabhakar integral and HilferPrabhakar fractional derivative. Even this formula is useful to solve problems associated with Hilfer-Prabhakar fractional derivative and its regularized version in engineering and mathematics.

Lemma 2 The Elzaki transform of Hilfer-Prabhakar derivative of fractional order (7) is given by

$$
\begin{aligned}
& \boldsymbol{E}\left(E_{\rho, v(1-\mu), \omega, 0^{+}}^{-\gamma v} \frac{d}{d t}\left(E_{\rho,(1-\gamma)(1-\mu), \omega, 0^{+}}^{-\gamma(1-\gamma)} g\right)\right)(p) \\
& =p^{-\mu}\left[1-\omega p^{\rho}\right]^{\gamma} \mathbf{E}[g](p)-p^{v(1-\mu)+1}\left[1-\omega p^{\rho}\right]^{\gamma \gamma}\left[E_{\rho,(1-\gamma)(1-\mu), \omega, 0^{+}}^{-\gamma(1-\gamma)} g(t)\right]_{t=0^{+}}
\end{aligned}
$$

Proof. Elzaki transform of Hilfer-Prabhakar fractional derivative using (5), (7) and convolution theorem for Elzaki transform, we have

$$
\begin{aligned}
\mathbf{E}\left(D_{\rho, \omega, 0^{+}}^{\gamma, \mu, v} g(t)\right)(p)= & \frac{1}{p} \cdot \mathbf{E}\left[t^{v(1-\mu)-1} E_{\rho, v(1-\mu)}^{-\gamma v}\left(\omega t^{\rho}\right)\right](p) \\
& \cdot \mathbf{E}\left[\frac{d}{d t}\left(E_{\rho,(1-\gamma)(1-\mu), \omega, 0^{+}}^{-\gamma(1-\gamma)} g\right)\right](p) \\
= & p^{v(1-\mu)}\left(1-\omega p^{\rho}\right)^{\gamma v} \cdot \mathbf{E}\left[\frac{d}{d t}\left(E_{\rho,(1-v)(1-\mu), \omega, 0^{+}}^{-\gamma(1-\gamma)} g\right)\right](p) \\
= & p^{\nu(1-\mu)}\left(1-\omega p^{\rho}\right)^{\gamma v}\left[p^{\gamma \mu-\nu-\mu}\left(1-\omega p^{\rho}\right)^{\gamma(1-v)} \mathbf{E}[g](p)\right.
\end{aligned}
$$




$$
\left.-p\left(E_{\rho,(1-\gamma)(1-\mu), \omega, 0^{+}}^{-\gamma(1-\gamma)} g\right)_{t=0^{+}}\right] .
$$

On simplification, we get the required result (11).

Lemma 3 The Elzaki transform of regularized version of Hilfer-Prabhakar fractional derivative (8) of order $\mu$ is expressed as

$$
\mathbf{E}\left[{ }^{C} D_{\rho, \omega, 0^{+}}^{\gamma, \mu} g\right](p)=p^{-\mu}\left(1-\omega p^{\rho}\right)^{\gamma} \mathbf{E}[g](p)-p^{2-\mu}\left[1-\omega p^{\rho}\right]^{\gamma} g\left(0^{+}\right) .
$$

Proof. Elzaki transform of regularized version of Hilfer-Prabhakar fractional derivative of order $\mu$ using (5) and (8), we have

$$
\begin{aligned}
\left.\mathbf{E}^{[}{ }^{C} D_{\rho, \omega, 0^{+}}^{\gamma, \mu} g\right](p) & =\mathbf{E}\left(E_{\rho, v(1-\mu), \omega, 0^{+}}^{-\gamma v} E_{\rho,(1-v)(1-\mu), \omega, 0^{+}}^{-\gamma(1-v)} \frac{d}{d t} g\right)(p) \\
& =\mathbf{E}\left(E_{\rho,(1-\mu), \omega, 0^{+}}^{-\gamma} \frac{d}{d t} g\right)(p) \\
& =\frac{1}{p} \cdot \mathbf{E}\left[t^{(1-\mu)-1} E_{\rho,(1-\mu)}^{-\gamma}\left(\omega t^{\rho}\right)\right](p) \cdot \mathbf{E}\left[\frac{d}{d t} g\right](p) \\
& =p^{-\mu}\left(1-\omega p^{\rho}\right)^{\gamma} \mathbf{E}[g](p)-p^{2-\mu}\left[1-\omega p^{\rho}\right]^{\gamma} g\left(0^{+}\right) .
\end{aligned}
$$

\section{Applications}

In this section, we discuss generalized fractional Free Electron Laser (FEL) equation which converge to FEL integro-differential equation,

$$
\left\{\begin{array}{l}
\frac{d y}{d x}=-i \pi g \int_{0}^{x}(x-t) e^{-i \eta(x-t)} y(t), g, \eta \in \mathbb{R}, x \in(0,1] \\
y(0)=1
\end{array}\right.
$$

Theorem 1 The solution of generalization the Free Electron Laser equation involving Hilfer-Prabhakar derivative [1]

$$
\left\{\begin{array}{l}
D_{\rho, \omega, 0^{+}}^{\gamma, \mu} y(x)=\gamma E_{\rho, \mu, \omega, 0^{+}}^{\beta} y(x)+g(x), x \in[0, \infty), \\
{\left[E_{\rho,(1-v)(1-\mu), \omega, 0^{+}}^{\gamma(x-1)} y(x)\right]_{t=0}=c, c \geq 0,} \\
\lim _{x \rightarrow \pm \infty} u(x, t)=0,
\end{array}\right.
$$


with $\mu \in(0,1), v \in[0,1], \omega \in \mathbb{R}, \rho>0, \gamma, \beta \geq 0$, is given by

$$
\begin{aligned}
y(p)= & \sum_{n=0}^{\infty}\left[c \cdot(\gamma)^{n} t^{\gamma(1-\mu)+\mu+2 \mu n-1} E_{\rho, v(1-\mu)+\mu(2 n+1)}^{(\beta+\gamma) n-\gamma(\nu-1)}\left(\omega t^{\rho}\right)\right. \\
& \left.+(\gamma)^{n^{\mu}} t^{\mu(2 n+1)-1} E_{\rho, \mu(2 n+1)}^{\gamma(n+1)+\beta n}\left(\omega t^{\rho}\right) \cdot g\right] .
\end{aligned}
$$

Proof. Taking Elzaki transform of (14) using (11), we have

$$
\begin{aligned}
& p^{-\mu}\left(1-\omega p^{\rho}\right)^{\gamma} \mathbf{E}[y](p)-p^{\nu(1-\mu)+1}\left(1-\omega p^{\rho}\right)^{\gamma \gamma} \cdot c \\
& =\gamma \cdot p^{\mu}\left(1-\omega p^{\rho}\right)^{-\beta} \mathbf{E}[y](p)+\mathbf{E}[g](p)
\end{aligned}
$$

$$
\begin{aligned}
& \mathbf{E}[y](p)=\frac{p^{\gamma(1-\mu)+1}\left(1-\omega p^{\rho}\right)^{\gamma \nu} \cdot c+\mathbf{E}[g](p)}{p^{-\mu}\left(1-\omega p^{\rho}\right)^{\gamma}-\gamma \cdot p^{\mu}\left(1-\omega p^{\rho}\right)^{-\beta}} \\
& =\frac{p^{\nu(1-\mu)+\mu+1}\left(1-\omega p^{\rho}\right)^{\gamma \nu-\gamma} \cdot c+p^{\mu}\left(1-\omega p^{\rho}\right)^{-\gamma} \mathbf{E}[g](p)}{1-\gamma \cdot p^{2 \mu}\left(1-\omega p^{\rho}\right)^{-(\beta+\gamma)}} \\
& =\sum_{n=0}^{\infty} \gamma^{n} c \cdot p^{\nu(1-\mu)+\mu+2 \mu n+1}\left(1-\omega p^{\rho}\right)^{\gamma(\nu-1)-(\beta+\gamma) n} \\
& +\sum_{n=0}^{\infty} \gamma^{n} p^{\mu+2 \mu n}\left(1-\omega p^{\rho}\right)^{-[\gamma+(\beta+\gamma) n]} \mathbf{E}[g],
\end{aligned}
$$

inverting Elzaki transform, we have

$$
\begin{aligned}
y(p)= & \sum_{n=0}^{\infty}\left[c \cdot \gamma^{n} t^{\gamma(1-\mu)+\mu+2 \mu n-1} E_{\rho, v(1-\mu)+\mu(2 n+1)}^{(\beta+\gamma) n-\gamma(v-1)}\left(\omega t^{\rho}\right)\right. \\
& \left.+\gamma^{n} t^{\mu(2 \mu+1)-1} E_{\rho, \mu(2 n+1)}^{\gamma(n+1)+\beta n}\left(\omega t^{\rho}\right) \cdot g\right] .
\end{aligned}
$$

Which is required solution.

Theorem 2 The solution to the Cauchy problem [5] of fractional order

$$
\left\{\begin{array}{l}
D_{\rho, \omega, 0^{+}}^{\gamma, \mu, v} u(x, t)=K \frac{\partial^{2}}{\partial x^{2}} u(x, t), \quad t>0, x \in \mathbb{R}, \\
{\left[E_{\rho,(1-v)(1-\mu), \omega, 0^{+}}^{-\gamma(1-v)} u(x, t)\right]_{t=0}=g(x),} \\
\lim _{x \rightarrow \pm \infty} u(x, t)=0,
\end{array}\right.
$$


with $\mu \in(0,1), v \in[0,1], \omega \in \mathbb{R}, \mathrm{K}, \quad \rho>0, \gamma \geq 0$, is given by $u(x, t)=\sum_{n=0}^{\infty} \frac{1}{2 \pi} \int_{-\infty}^{\infty}\left(-K k^{2}\right)^{n} e^{i k x} \hat{g}(k) d k t^{v(1-\mu)+\mu(n+1)-1} E_{\rho, v(1-\mu)+\mu(n+1)}^{\gamma[n-(v-1)]}\left(\omega t^{\rho}\right)$.

Proof. Let $\bar{u}(x, p)=\mathbf{E}(u)(x, p)$ and $\hat{u}(k, t)=F(u)(k, t)$ are the Elzaki transform and the Fourier transform respectively. Taking Elzaki transform of (15) and by using (11), we have

$$
\begin{aligned}
& p^{-\mu}\left(1-\omega p^{\rho}\right)^{\gamma} \widehat{\bar{u}}(k, p)-p^{\nu(1-\mu)+1}\left(1-\omega p^{\rho}\right)^{\gamma \gamma} \widehat{g}(k)=-K k^{2} \widehat{\bar{u}}(k, p) \\
& \widehat{\bar{u}}(k, p)=\frac{p^{v(1-\mu)+1}\left(1-\omega p^{\rho}\right)^{\gamma v} \hat{g}(k)}{p^{-\mu}\left(1-\omega p^{\rho}\right)^{\gamma}+K k^{2}} \\
& =\frac{p^{v(1-\mu)+1+\mu}\left(1-\omega p^{\rho}\right)^{\gamma(\nu-1)} \hat{g}(k)}{1+\frac{K k^{2}}{p^{-\mu}\left(1-\omega p^{\rho}\right)^{\gamma}}} \\
& =\sum_{n=0}^{\infty}\left(-K k^{2}\right)^{n} \widehat{g}(k) p^{v(1-\mu)+\mu(n+1)+1}\left(1-\omega p^{\rho}\right)^{\gamma[(v-1)-n]} \text {, }
\end{aligned}
$$

inverting Elzaki transform, we have

$$
\hat{u}(k, t)=\sum_{n=0}^{\infty}\left(-K k^{2}\right)^{n} \hat{g}(k) t^{v(1-\mu)+\mu(n+1)-1} E_{\rho, v(1-\mu)+\mu(n+1)}^{\gamma[n-(v-1)]}\left(\omega t^{\rho}\right) .
$$

inverting Fourier transform and after little simplification, finally we obtain the desired result.

Theorem 3 The solution of the Cauchy problem [5] of fractional order

$$
\left\{\begin{array}{l}
\mathbf{c}_{\mathbf{D}_{\rho, \omega, 0^{+}}^{\gamma}}^{\gamma, \mu} \mathbf{u}(x, t)=K \frac{\partial^{2}}{\partial x^{2}} \mathrm{u}(x, t), \quad t>0, x \in \mathbb{R} \\
u\left(x, 0^{+}\right)=g(x) \\
\lim _{x \rightarrow \pm \infty} u(x, t)=0
\end{array}\right.
$$

with $\mu \in(0,1), v \in[0,1], \omega \in \mathbb{R}, K, \rho>0, \gamma \geq 0$, is given by

$$
u(x, t)=\sum_{n=0}^{\infty} \frac{1}{2 \pi} \int_{-\infty}^{\infty}\left(-K k^{2}\right)^{n} e^{i k x} \hat{g}(k) d k t^{\mu n} E_{\rho, \mu n+1}^{\gamma n}\left(\omega t^{\rho}\right) .
$$


Proof. Taking Elzaki-Fourier transform of (17) using (12), we have

$$
\begin{aligned}
& p^{-\mu}\left(1-\omega p^{\rho}\right)^{\gamma} \widehat{\bar{u}}(k, p)-p^{2-\mu}\left(1-\omega p^{\rho}\right)^{\gamma} \hat{g}(k)=-K k^{2} \widehat{\bar{u}}(k, p) \\
& \widehat{\bar{u}}(k, s)=\frac{p^{2-\mu}\left(1-\omega p^{\rho}\right)^{\gamma} \hat{g}(k)}{p^{-\mu}\left(1-\omega p^{\rho}\right)^{\gamma}+K k^{2}} \\
&=\frac{p^{2} \hat{g}(k)}{1+\frac{K k^{2}}{p^{-\mu}\left(1-\omega p^{\rho}\right)^{\gamma}}} \\
&=\sum_{n=0}^{\infty}\left(-K k^{2}\right)^{n} \hat{g}(k) p^{\mu n+2}\left(1-\omega p^{\rho}\right)^{-\gamma n},
\end{aligned}
$$

inverting Elzaki transform, we have

$$
\widehat{u}(k, t)=\sum_{n=0}^{\infty}\left(-K k^{2}\right)^{n} \hat{g}(k) t^{\mu n} E_{\rho, \mu n+1}^{\gamma n}\left(\omega t^{\rho}\right),
$$

inverting Fourier transform, we have

$$
u(x, t)=\sum_{n=0}^{\infty} \frac{1}{2 \pi} \int_{-\infty}^{\infty}\left(-K k^{2}\right)^{n} e^{i k x} \hat{g}(k) d k t^{\mu n} E_{\rho, \mu n+1}^{\gamma n}
$$

which is the claimed result.

\section{Conclusions}

In this work, we studied the Elzaki transform of Prabhakar integral plays a key role to control engineering calculus problem in fractional related to HilferPrabhakar fractional derivative and its regularized version. We also present some application of Hilfer-Prabhakar and its regularized version along with the integral transform mainly Elzaki transform. In this paper we consider Free Electron Laser problem. Furthermore, we discuss some application of Elzaki transform to find solution of Cauchy problem involving Hilfer-Prabhakar fractional derivative and its regularized version. We also find the point where the very popular Laplace transform and the new Elzaki transform coincide. The results show that Elzaki transform is very useful for solving fractional differential equations. 


\section{References}

[1] G. Dattoli, L. Gianessi, L. Mezi, D. Tocci , R. Colai, FEL time-evolution operator, Nucl. Instr. Methods A, 304 (1991), 541-544.

[2] Tarig. M. Elzaki, The New Integral Transform "Elzaki Transform" fundamental properties investigations and applications, GJPAM, 7 (1) (2011), $57-64$.

[3] Tarig M. Elzaki, Application of New Transform "Elzaki Transform" to Partial Differential Equations, GJPAM, 7 (1) (2011), 65-70.

[4] M. Eslaminasab, S. Abbasbandy, Study on usage of Elzaki transform for the ordinary differential equations with non-constant coefficients, Int. J. Ind. Math., 7 (3) 2015, Article ID IJIM-00670 (5 pages).

[5] R. Garra, R. Goreno, F. Polito, Z. Tomovski, Hilfer-Prabhakar Derivatives and Some Applications, Appl. Math. Comput., 242 (1) (2014), 576-589.

[6] R. Gorenflo, F. Mainardi, Fractional calculus: integral and differential equations of fractional order, In A. Carpinteri and F. Mainardi Fractals and Fractional Calculus in Continuum Mechanics, Springer-Verlag, Wien, (1997), 223-276.

[7] R. Hilfer, Fractional calculus and regular variation in thermodynamics, In R. Hilfer, editor, Applications of Fractional Calculus in Physics, 429, Singapore, 2000. World Scientific.

[8] A. A. Kilbas, M. Saigo, R. K. Saxena, Generalized Mittag-Leffler function and generalized fractional calculus operators, Integral Transform Spec. Funct., 15 (1) (2004), 31-49.

[9] A. A. Kilbas, H. M. Srivastava, J. J. Trujillo, Theory and Applications of the Fractional Differential Equations, North-Holland Math. Stud., 204 (2006).

[10] D. Kumar, J. Singh, M. A. Qurashi, D. Baleanu, Analysis of Logistic Equation pertaining to a new fractional derivative with non-singular kernel, Adv. Mech. Eng., 9 (2) (2017), 1-8.

[11] D. Kumar, J. Singh, D. Baleanu, A new fractional model for convective straight fins with temperature-dependent thermal conductivity, Thermal Sci., 22 (6B) (2018), 2791-2802. 
[12] I. Podlubny, Fractional Differential Equations, Academic Press, New York, 1999.

[13] Y. Z. Povstenko, Evolution of the initial box-signal for time-fractional diffusion-wave equation in a case of different spatial dimensions, Physica A, 389 (1) (2010), 4696-4707.

[14] T. R. Prabhakar, A singular integral equation with a generalized MittagLeffler function in the kernel, Yokohama Math. J., 19 (1971), 7-15.

[15] S. G. Samko, A. A. Kilbas, O. I. Marichev, Fractional integrals and derivatives, Gordon and Breach Science, Yverdon, 1993.

[16] T. Sandev, R. Metzler, Z. Tomovski, Fractional diffusion equation with a generalized Riemann-Liouville time fractional derivative, J. Phys. A, 44 (25) (2011), 255203.

[17] J. Singh, D. Kumar, J. J. Nieto, Analysis of an El Nino Southern Oscillation model with a new fractional derivative, Chaos Solitons Fractals, 99 (2017), 109-115.

[18] J. Singh, D. Kumar, D. Baleanu, Analysis of a new fractional model for damped Burger equations, Open Phys., 15 (2017), 35-41.

[19] H. M. Srivastava, Z. Tomovski, Fractional calculus with an integral operator containing a generalized Mittag-Leffler function in the kernel, Appl. Math.Comput., 211 (1) (2009), 198-210.

[20] Z. Tomovski, R. Hilfer, H. M. Srivastava,Fractional and operational calculus with generalized fractional derivative operators and Mittag-Leffler type functions, Integral Transform Spec. Funct., 21 (11) (2010), 797-814.

[21] Y. Zhou, F. Jiao, Nonlocal Cauchy problem for fractional evolution equations, Nonlinear Anal Real World Appl., 11 (5) (2010), 4465-4475. 